\title{
Synthesis and structural characterization of products condensation 4-carboxy-1-(4-styrylcarbonylphenyl)-2-pyrrolidinones with hydrazines
}

\author{
Ausra Voskiene, ${ }^{a}$ Vytautas Mickevicius, ${ }^{a}$ and Gema Mikulskiene ${ }^{b_{*}}$ \\ ${ }^{a}$ Department of Organic Chemistry, Kaunas University of Technology, Radvilenu pl. 19, \\ LT-50254, Kaunas, Lithuania \\ ${ }^{b}$ Department of Bioorganic Compounds Chemistry, Institute of Biochemistry, Mokslininku str.12, \\ LT-08662, Vilnius, Lithuania \\ E-mail: gemam@bchi.lt
}

\begin{abstract}
The synthesis of some new chalcones is described. A series of the pyrazole and pyrazoline type derivatives were obtained by the condensation of the chalcones with hydrazine and phenylhydrazine. All compounds were characterized using NMR, IR, MS techniques. Computer molecular modeling assisted for NMR spectral analysis.
\end{abstract}

Keywords: Pyrrolidinones, chalcones, pyrazolines, pyrazoles, NMR

\section{Introduction}

The chalcones are the convenient intermediates for the synthesis five, ${ }^{[1,2]}$ six ${ }^{[1,3]}$ and seven members ${ }^{[4]}$ heterocycles, often have exhibited diverse biological activity. Some pyrazoline derivatives were used as the bacteriostatic, fungicidal, and anticancer agents. ${ }^{[5]} \mathrm{We}$ continued our interest in the chemistry of $\mathrm{N}$-substituted amino acids and the products of their cyclization, in this case - 1,3-disubstituted 2-pyrrolidinones. The structure of newly synthesized compounds was mainly characterized by NMR spectroscopy. The influence of the 5-substituent on the carbons and protons of pyrazoline and pyrazole derivatives has been discussed. The substitution of benzene ring as the starting chalcones 3a-c, ${ }^{[6-8]}$ as the pyrazoline ${ }^{[2,9-18]}$ and pyrazole ${ }^{[19-34]}$ derivatives was taken into account. The average increments of pyrazoline and pyrazole for aromatic carbon atoms as for monosubstituted benzene rings were determined. The calculated extended Hückel charges followed the carbons chemical shifts verify the assignment. 


\section{Results and Discussion}

The starting product - 1-(4-acetylphenyl)-4-carboxy-2-pyrrolidinone $\mathbf{2}$ was synthesized according to the known method by the refluxing 4-aminoacetophenone 1 with itaconic acid in water. The chalcones 3a-c were obtained in good yields by base $(\mathrm{NaOH})$ catalyzed aldol condensation of the substituted acetophenone derivative $\mathbf{2}$ and the substituted benzaldehydes in ethanol (Scheme 1).

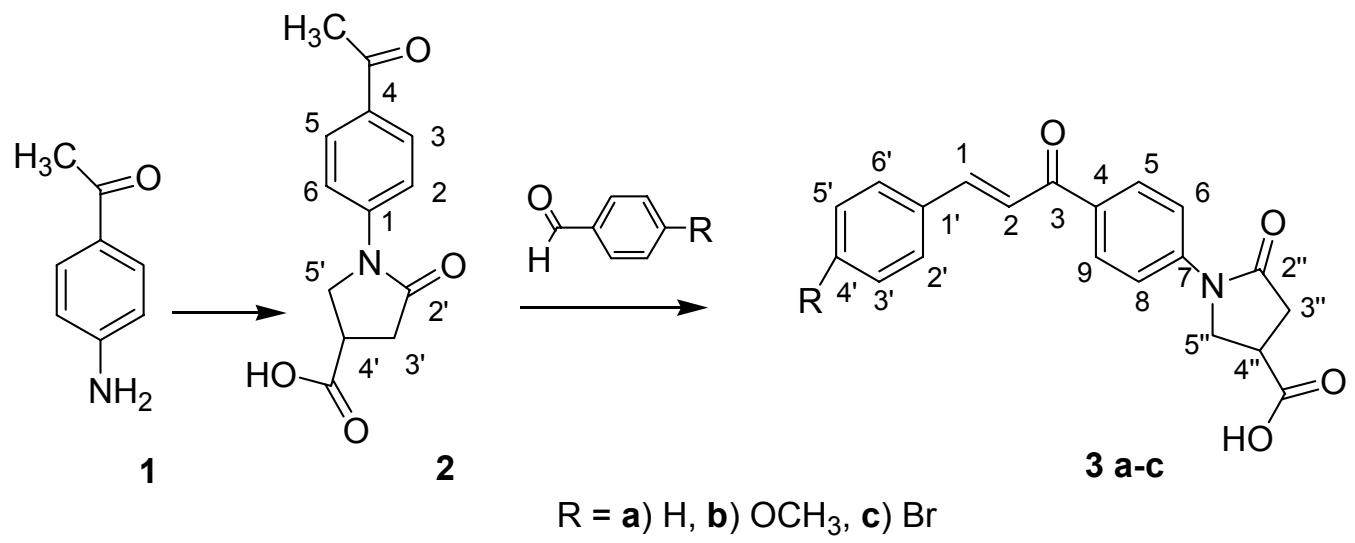

Scheme 1. Synthesis of 4-carboxy-1-(4-styrylcarbonylphenyl)-2-pyrrolidinones.

Compounds 3a-c were tested as precursors in the synthesis of the pyrazole and the pyrazoline derivatives (Scheme 2) by reaction with hydrazine hydrate or phenylhydrazine.

The reactions between the chalcones 3a-c and the hydrazine hydrate in refluxing ethanol and the acetic acid was monitored by TLC. After 5 hours all starting material was consumed. Condensation of the chalcones 3a-c and the hydrazine hydrate in ethanol gave the corresponding 1-[4-(5-aryl-4,5-dihydro-1H-pyrazol-3-yl)phenyl]-5-oxo-pyrrolidine-3-carboxylic acids 4a-c, while in acetic acid - 1-[4-(1-acetyl-5-aryl-4,5-dihydro-1H-pyrazol-3-yl)phenyl]-5oxopyrrolidine-3-carboxylic acids 5a-c were formed. The chalcones 3a-c reacted with phenylhydrazine in boiling 1,4-dioxane resulting in the formation of 1-[4-(1,5-diaryl-4,5dihydro-1H-pyrazol-3-yl)phenyl]-5-oxopyrrolidine-3-carboxylic acids 6a-c, while in acetic acid - pyrazole derivatives $7 \mathbf{a , b}$.

The structure of the above mentioned compounds was confirmed by NMR, IR and MS spectral data. The most comprehensive analytical data were obtained by NMR studies. The assignment was made on the substituents additivity rules, spectral characteristics of structurally related compounds, signal intensities and multiplicities. An APT ${ }^{13} \mathrm{C}$ NMR experiment was used to prove the interpretation of the carbon resonances in some cases. The data on ${ }^{1} \mathrm{H}$ and ${ }^{13} \mathrm{C}$ NMR chemical shifts are presented in experimental part. The carbon atoms are marked arbitrary according to the numbering given in Scheme 1 and Scheme 2. 

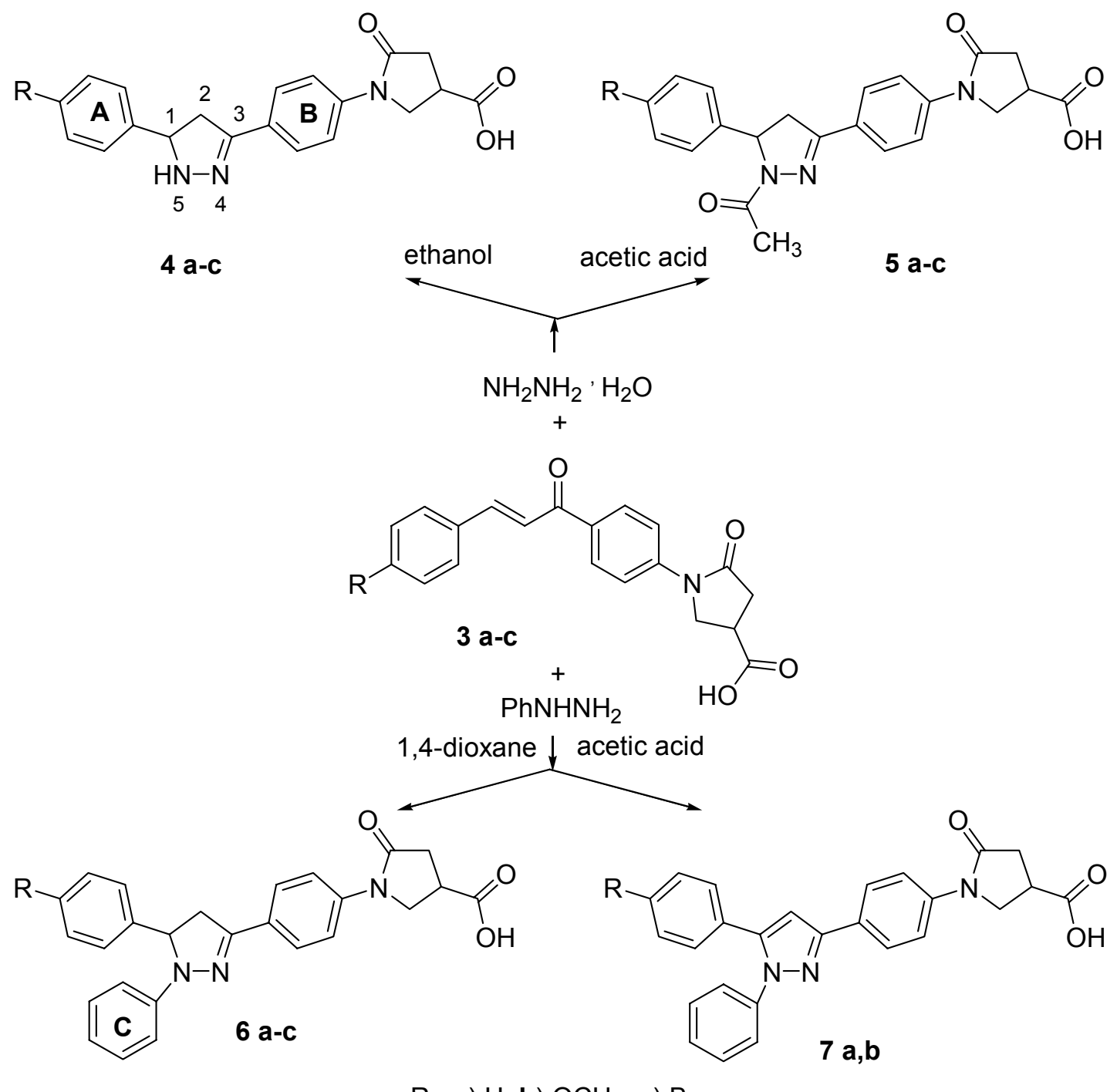

$\mathrm{R}=\mathbf{a}) \mathrm{H}$, b) $\left.\mathrm{OCH}_{3}, \mathbf{c}\right) \mathrm{Br}$

Scheme 2. Synthesis of substituted pyrazoline and pyrazole derivatives.

The studied compounds possess nonsubstituted (a), $\mathrm{OCH}_{3}$ (b) and $\mathrm{Br}$ (c) aromatic derivatives. Due to the substituent $\mathrm{OCH}_{3}{ }^{13} \mathrm{C}$ NMR chemical shift of $\mathrm{C}-2$ atom in compound $\mathbf{3 b}$ is shielded $2.5 \mathrm{ppm}$, while the atoms $\mathrm{C}-1$ and C-3 were much less shielded $(0.2 \mathrm{ppm}$ and 0.1 $\mathrm{ppm})$. Due to the $\mathrm{Br}$ substitution observed the low shielding effect $1.0 \mathrm{ppm}$ for the $\mathrm{C}-1$, and deshielding $0.7 \mathrm{ppm}$. for the $\mathrm{C}-2$ atom. ${ }^{1} \mathrm{H}$ NMR spectra showed a weak shielding for $\mathrm{H}-1$ and $\mathrm{H}-$ 2 protons, especially for the $\mathrm{H}-2$ proton $(0.17 \mathrm{ppm})$ due to the $\mathrm{Br}$ substitution.

Values of $63.7 \mathrm{ppm}, 40.6 \mathrm{ppm}$ and $148.3 \mathrm{ppm}$ in ${ }^{13} \mathrm{C}$ NMR spectra of compounds 4a-c confirmed formation of the pyrazoline fragment after the condensation of the compounds 3a-c with the hydrazine hydrate in ethanol. Substitution of the aromatic ring $\left(\mathrm{OCH}_{3}, \mathrm{Br}\right)$ caused a low shielding effect for all carbon atoms of the pyrazoline moiety, only C-3 was negligibly deshielded. In the ${ }^{1} \mathrm{H}$ NMR spectra the 5-membered heterocyclic derivatives are observed as 
ABX type spin system pattern. Observed downfield chemical shift values of all the pyrrolidinone carbon atoms and the 4"'-COOH carbon were caused by the interactions of the unsubstituted $\mathrm{NH}$ hydrogen with $\mathrm{CO}$ groups.

The spectra of the 5-membered heterocycles in compounds 5a-c and 6a-c changed significantly when substituents $\mathrm{COCH}_{3}$ and $\mathrm{Ph}$ are attached in position 5. The substituent $\mathrm{COCH}_{3}$ influenced C-1, C-2 and C-3 atoms about - $4.2 \mathrm{ppm}, 1.4 \mathrm{ppm}$ and $5.3 \mathrm{ppm}$ respectively. By the ascendancy of the substituent $\mathrm{Ph}$ the atoms of 5-membered heterocycle $\mathrm{C}-1$ and $\mathrm{C}-3$ is shifted upfield about $-0.6 \mathrm{ppm}$ and $4.1 \mathrm{ppm}, \mathrm{C}-2-$ downfield up to $2.8 \mathrm{ppm}$.

The 5-membered heterocycle pyrazole in compounds $7 \mathbf{a}, \mathbf{b}$ is characteristically influenced by phenyl substituents. The C-3 atom is found about $144.0 \mathrm{ppm}$, as in case of compounds 6a-c, while $\mathrm{C}-1$ and $\mathrm{C}-2$ are observed at $\sim 150.5 \mathrm{ppm}$ and $\sim 104.9 \mathrm{ppm}$.

The average increments of the pyrazoline and the pyrazole for aromatic carbon atoms in 1,3,5-positions (A, B, C benzene rings) as for monosubstituted benzene rings were determined after assignment of all carbons of the studied compounds (Table 1). Values of the influences of $\mathrm{CH}=\mathrm{CH}-\mathrm{CO}$ fragment for $\mathbf{A}$ and $\mathbf{B}$ monosubstituted aromatic rings found in literature were verified. The increments of the pyrrolidinone ring, used in this calculation were found in previous our work. ${ }^{[34]}$

Table 1. The average increments of pyrazoline and pyrazole for chemical shifts of monosubstituted benzene carbons of the studied compounds

\begin{tabular}{|c|c|c|c|c|c|c|}
\hline \multirow{2}{*}{ 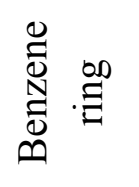 } & \multirow{2}{*}{ Substitution } & \multicolumn{5}{|c|}{ Class of compounds } \\
\hline & & 3 & 4 & 5 & 6 & 7 \\
\hline \multirow{4}{*}{$\stackrel{\infty 0}{\Xi}$} & $\mathrm{C}-i$ & 6.7 & 14.4 & 14.2 & 14.3 & 1.5 \\
\hline & $\mathrm{C}-\mathrm{o}$ & 0.8 & -1.6 & -2.4 & -1.8 & 0.2 \\
\hline & $\mathrm{C}-m$ & 0.3 & -0.3 & -0.1 & 0.2 & -0.1 \\
\hline & $\mathrm{C}-p$ & 1.6 & -1.4 & -1.4 & -1.3 & -0.2 \\
\hline \multirow{4}{*}{ 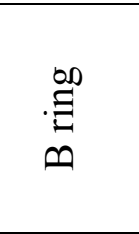 } & $\mathrm{C}-i$ & 8.8 & 3.6 & 2.4 & 3.8 & 3.7 \\
\hline & $\mathrm{C}-\mathrm{o}$ & 0.9 & -2.8 & -1.4 & -2.4 & -3.0 \\
\hline & $\mathrm{C}-m$ & -0.9 & -0.5 & -0.4 & -0.1 & -0.0 \\
\hline & C- $p$ & 4.3 & 0.1 & 1.5 & 0.3 & -0.3 \\
\hline \multirow{4}{*}{ U } & $\mathrm{C}-i$ & - & - & - & 18.4 & 11.3 \\
\hline & $\mathrm{C}-o$ & - & - & - & -15.6 & -3.2 \\
\hline & $\mathrm{C}-m$ & - & - & - & 0.4 & 0.6 \\
\hline & C- $p$ & - & - & - & -9.8 & -0.1 \\
\hline
\end{tabular}

Investigation of molecular structure of the studied compounds by the computer molecular modeling showed that all molecules are near to planar. Calculated Hückel charges successfully followed the chemical shifts of the studied compounds in ${ }^{13} \mathrm{C}$ NMR spectra (Table 2). 
The charge allocation obtained by MM2 method for the optimized molecules models of studied compounds afforded ground for calculating the $\pi$-bond orders other than $1,1.5,2$. The analysis of this computation showed the formation of the wide-ranging extended $\pi$-system in the molecules of compounds 3a-c, 7a,b (Table 3).

Table 2. ${ }^{13} \mathrm{C}$ NMR chemical shifts of $\mathrm{C}-1, \mathrm{C}-2, \mathrm{C}-3$ atoms of compounds 3a-c, 5a-c and corresponding Hückel charges

\begin{tabular}{ccccc}
\hline \multirow{2}{*}{ Compound } & Carbon atom & $\mathbf{a}$ & $\mathbf{b}$ & $\mathbf{c}$ \\
\hline \multirow{3}{*}{3} & $\mathrm{C}-1$ & $143.2 / 0.053$ & $143.0 / 0.054$ & $142.2 / 0.047$ \\
& $\mathrm{C}-2$ & $121.9 /-0.093$ & $119.4 /-0.126$ & $122.7 /-0.129$ \\
& $\mathrm{C}-3$ & $187.7 / 0.398$ & $187.5 / 0.392$ & $187.5 / 0.386$ \\
\multirow{3}{*}{$\mathbf{5}$} & $\mathrm{C}-1$ & $59.4 / 0.043$ & $58.9 / 0.042$ & $58.8 / 0.042$ \\
& $\mathrm{C}-2$ & $42.1 /-0.090$ & $42.1 /-0.094$ & $41.9 /-0.096$ \\
& $\mathrm{C}-3$ & $153.7 / 0.083$ & $153.7 / 0.084$ & $153.7 / 0.085$ \\
\hline
\end{tabular}

Table 3. $\pi$-Bond order, computed from molecular models of compounds 3a and 7a

\begin{tabular}{ccc}
\hline Bonds & $\mathbf{3 a}$ & $\mathbf{7 a}$ \\
\hline $\mathrm{C}(1 ')-\mathrm{C}(1)$ & 1.272 & 1.164 \\
$\mathrm{C}(1)-\mathrm{C}(2)$ & 1.924 & 1.729 \\
$\mathrm{C}(2)-\mathrm{C}(3)$ & 1.261 & 1.580 \\
$\mathrm{C}(3)-\mathrm{C}(4)$ & 1.224 & 1.245 \\
$\mathrm{~N}(4)-\mathrm{N}(5)$ & - & 1.373 \\
$\mathrm{~N}(5)-\mathrm{C}(10)$ & - & 1.277 \\
$\mathrm{C}(3)-\mathrm{N}(4)$ & - & 1.700 \\
$\mathrm{C}(7)-\mathrm{N}(1)$ & 1.278 & 1.268 \\
$\mathrm{~N}(1)-\mathrm{C}\left(2^{\prime \prime}\right)$ & 1.454 & 1.456 \\
$\mathrm{C}\left(2^{\prime \prime}\right)-\mathrm{O}$ & 1.796 & 1.794 \\
$\mathrm{C}\left(2^{\prime \prime}\right)-\mathrm{C}\left(3^{\prime \prime}\right)$ & 1.000 & 1.000 \\
$\mathrm{~N}(1)-\mathrm{C}\left(5^{\prime \prime}\right)$ & 1.000 & 1.000 \\
\hline
\end{tabular}

\section{Experimental Section}

General Procedures. The ${ }^{1} \mathrm{H}$ and ${ }^{13} \mathrm{C}$ spectra were recorded on a Varian Unity Inova $(300 \mathrm{MHz})$ spectrometer operating in the Fourier transform mode. Chemical shifts $(\delta)$ are given from TMS $(0.0 \mathrm{ppm})$ as an internal standard for ${ }^{1} \mathrm{H}$ NMR, and $\mathrm{d}_{6}$-DMSO (39.5 ppm) for ${ }^{13} \mathrm{C}$ NMR. Melting points were determined on an automatic APA1 melting point apparatus and are uncorrected. The IR spectra were determined in potassium bromide pellets on a Perkin - Elmer FT-IR system 
spectrum GX spectrometer. Mass spectra were obtained on a Waters (Micromass) ZQ 2000 spectrometer using chemical ionization (CI) mode. Elemental analyses were performed on a CE440 elemental analyser.

The molecular modeling of the study compounds was carried out using Chem 3D Ultra 9.0 (Licence Cambridge Software Package, Serial number: 031406391 4800).

1-(4-Acetylphenyl)-4-carboxy-2-pyrrolidinone (2). A mixture of $67.58 \mathrm{~g}$ (0.5 mol) 4aminoacetophenone 1 and $78.06 \mathrm{~g}(0.6 \mathrm{~mol})$ itaconic acid in $150 \mathrm{ml}$ of water was heated at reflux 15 hours, then treated with $280 \mathrm{ml} 10 \% \mathrm{NaOH}$. After cooling to $20^{\circ} \mathrm{C}$ the reaction mixture was filtered, filtrate acidified with aq $\mathrm{HCl}$ to $\mathrm{pH} 1$, resulting precipitate filtered, then washed with water, yield 84\%; mp 170-171 ${ }^{\circ} \mathrm{C}$ (2-propanol); IR (KBr), $\mathrm{cm}^{-1}: 3006(\mathrm{OH}), 1726,1702,1639$ $(\mathrm{C}=\mathrm{O}) . \mathrm{MS} \mathrm{m} / \mathrm{z},(\%):[\mathrm{M}+\mathrm{H}]^{+}=248.5(100) .{ }^{1} \mathrm{H}$ NMR (300 MHz, $\left.\mathrm{d}_{6}-\mathrm{DMSO}\right) \delta: 2.57(\mathrm{~s}, 3 \mathrm{H}$, $\left.\mathrm{CH}_{3} \mathrm{CO}\right), 2.70-2.87\left(\mathrm{~m}, 2 \mathrm{H}, \mathrm{CH}_{2} \mathrm{CO}\right), 3.31-3.43(\mathrm{~m}, 1 \mathrm{H}, \mathrm{CH}), 3.99-4.13\left(\mathrm{~m}, 2 \mathrm{H}, \mathrm{CH}_{2} \mathrm{~N}\right)$, 7.81, $7.96\left(2 \mathrm{~d}, J=9.0 \mathrm{~Hz}, 4 \mathrm{H}, \mathrm{H}_{\mathrm{ar}}\right), 12.86(\mathrm{~s}, 1 \mathrm{H}, \mathrm{COOH}) .{ }^{13} \mathrm{C}$ NMR $\left(75 \mathrm{MHz}, \mathrm{d}_{6}\right.$-DMSO) $\delta: 26.5$ $\left(\mathrm{COCH}_{3}\right), 35.0$ (C-4), 35.3 (C-3), 49.7 (C-3), 118.4 (C-2', 6'), 129.1 (C-3', 5'), 132.0 (C-4'), $143.1\left(\mathrm{C}-1\right.$ '), $172.6(\mathrm{COOH}), 174.0(\mathrm{C}-2), 196.6\left(\mathrm{COCH}_{3}\right)$. Anal. calcd. for $\mathrm{C}_{13} \mathrm{H}_{13} \mathrm{NO}_{4},(\%): \mathrm{C}$ 63.15, H 5.30, N 5.67; Found (\%), C 62.48, H 4.89, N 5.29.

4-Carboxy-1-(4-styrylcarbonylphenyl)-2-pyrrolidinones (3a-c). General Procedure. A mixture $2.47 \mathrm{~g}$ (0.01 mol) 1-(4-acetylphenyl)-4-carboxy-2-pyrrolidinone 2, $0.015 \mathrm{~mol}$ corresponding benzaldehide and $15 \mathrm{ml} 10 \% \mathrm{NaOH}$ in $10 \mathrm{ml}$ of ethanol was refluxed for 4 hours and cooled, $15 \mathrm{ml}$ water was added and acidified with aq $\mathrm{HCl}$ to $\mathrm{pH} 1-2$. The precipitate filtered, and then washed with water.

4-Carboxy-1-(4-styrylcarbonylphenyl)-2-pyrrolidinone (3a). Yield $74 \%, \mathrm{mp} \quad 208-209^{\circ} \mathrm{C}$ (ethanol), IR (KBr), cm ${ }^{-1}: 3446(\mathrm{OH}), 1726,1694,1660(\mathrm{C}=\mathrm{O}), 1602(\mathrm{CH}=\mathrm{CH}) . \mathrm{MS} \mathrm{m} / \mathrm{z}$, $(\%):[\mathrm{M}+\mathrm{H}]^{+}=336.4(100) .{ }^{1} \mathrm{H}$ NMR $\left(300 \mathrm{MHz}, \mathrm{d}_{6}-\mathrm{DMSO}\right) \delta: 2.72-2.89\left(\mathrm{~m}, 2 \mathrm{H}, \mathrm{CH}_{2} \mathrm{CO}\right), 3.34-$ $3.46(\mathrm{~m}, 1 \mathrm{H}, \mathrm{CH}), 4.03-4.17\left(\mathrm{~m}, 2 \mathrm{H}, \mathrm{CH}_{2} \mathrm{~N}\right), 7.46-7.51\left(\mathrm{~m}, 3 \mathrm{H}, \mathrm{H}_{\mathrm{ar}}\right), 7.76(\mathrm{~d}, J=15.6 \mathrm{~Hz}, 1 \mathrm{H}$, $(\underline{\mathrm{CH}}=\mathrm{CH})), 7.87-7.98\left(\mathrm{~m}, 4 \mathrm{H}, \mathrm{H}_{\mathrm{ar}}\right), 7.99(\mathrm{~d}, J=15.6 \mathrm{~Hz}, 1 \mathrm{H},(\mathrm{CH}=\underline{\mathrm{CH}})), 8.23(\mathrm{~d}, 2 \mathrm{H}, J=8.9 \mathrm{~Hz}$, $\mathrm{H}_{\mathrm{ar}}$ ), 12.91 (s, 1H, COOH). ${ }^{13} \mathrm{C}$ NMR (75 MHz, d $\mathrm{d}_{6}$-DMSO) $\delta: 35.1$ (C-4"'), 35.4 (C-3"'), 49.8 (C-5'), 118.5 (C-6, 8), 121.9 (C-2), 128.9 (C-2', 6'), 128.9 (C-3', 5'), 129.5 (C-5, 9), 130.6 (C4'), 132.7 (C-4), 134.7 (C-1'), 143.2 (C-1), 143.6 (C-7), 172.7 (COOH), 174.1 (C-2'), 187.7 (C3). Anal. calcd. for $\mathrm{C}_{20} \mathrm{H}_{17} \mathrm{NO}_{4}$, (\%): C 71.63, H 5.11, N 4.18; Found (\%), C 71.2, H 5.50, N 3.9. 4-Carboxy-1-[4-(4-methoxystyrylcarbonyl)phenyl]-2-pyrrolidinone (3b). Yield 71\%, mp 197-198 ${ }^{\circ} \mathrm{C}$ (ethanol), IR (KBr), cm ${ }^{-1}: 3341(\mathrm{OH}), 1731,1711,1652(\mathrm{C}=\mathrm{O}), 1593(\mathrm{CH}=\mathrm{CH}) . \mathrm{MS}$ $\mathrm{m} / \mathrm{z},(\%):[\mathrm{M}+\mathrm{H}]^{+}=366.4(100) .{ }^{1} \mathrm{H}$ NMR $\left(300 \mathrm{MHz}, \mathrm{d}_{6}-\mathrm{DMSO}\right) \delta: 2.72-2.90\left(\mathrm{~m}, 2 \mathrm{H}, \mathrm{CH}_{2} \mathrm{CO}\right)$, 3.34-3.44 (m, 1H, CH), $3.82\left(\mathrm{~s}, 3 \mathrm{H}, \mathrm{OCH}_{3}\right), 4.02-4.16\left(\mathrm{~m}, 2 \mathrm{H}, \mathrm{CH}_{2} \mathrm{~N}\right), 7.02(\mathrm{~d}, J=8.8 \mathrm{~Hz}, 2 \mathrm{H}$, $\left.\mathrm{H}_{\mathrm{ar}}\right), 7.70(\mathrm{~d}, J=15.5 \mathrm{~Hz}, 1 \mathrm{H},(\underline{\mathrm{CH}}=\mathrm{CH})), 7.82(\mathrm{~d}, J=15.5 \mathrm{~Hz}, 1 \mathrm{H},(\mathrm{CH}=\underline{\mathrm{CH}})), 7.85(\mathrm{~d}, J=9.0 \mathrm{~Hz}$, $\left.2 \mathrm{H}, \mathrm{H}_{\mathrm{ar}}\right), 7.86\left(\mathrm{~d}, J=8.8 \mathrm{~Hz}, 2 \mathrm{H}, \mathrm{H}_{\mathrm{ar}}\right), 8.18\left(\mathrm{~d}, J=9.0 \mathrm{~Hz}, 2 \mathrm{H}, \mathrm{H}_{\mathrm{ar}}\right), 12.89(\mathrm{~s}, 1 \mathrm{H}, \mathrm{COOH}) .{ }^{13} \mathrm{C}$ NMR (75 MHz, d6-DMSO) 8: 35.1 (C-4'”), 35.4 (C-3'), 49.8 (C-5'), $55.4\left(\mathrm{OCH}_{3}\right), 114.4$ (C-3', 5'), 118.5 (C-6, 8), 119.4 (C-2), 127.4 (C-1'), 129.5 (C-5, 9), 130.8 (C-2', 6'), 133.0 (C-4), 143.0 
(C-1), 143.6 (C-7), 161.3 (C-4'), 174.1 (C-2'’), 172.6 (COOH), 187.5 (C-3). Anal. calcd. for $\mathrm{C}_{21} \mathrm{H}_{19} \mathrm{NO}_{5}$, (\%): C 69.03, H 5.24, N 3.83; Found (\%), C 65.9, H 5.17, N 3.70.

1-[4-(4-Bromostyrylcarbonyl)phenyl]-4-carboxy-2-pyrrolidinone (3c). Yield 78\%, mp 243$244^{\circ} \mathrm{C}$ (dioxane), IR (KBr), $\mathrm{cm}^{-1}: 3457(\mathrm{OH}), 1732,1661,1607(\mathrm{C}=\mathrm{O}), 1595(\mathrm{CH}=\mathrm{CH}) . \mathrm{MS} \mathrm{m} / \mathrm{z}$, (\%): $[\mathrm{M}+\mathrm{H}]^{+}=414.3(98),[\mathrm{M}+\mathrm{H}+2]^{+}=416.3(100) .{ }^{1} \mathrm{H} \mathrm{NMR}\left(300 \mathrm{MHz}, \mathrm{d}_{6}\right.$-DMSO) $\delta: 2.72-2.90$ (m, 2H, $\left.\mathrm{CH}_{2} \mathrm{CO}\right), 3.34-3.44(\mathrm{~m}, 1 \mathrm{H}, \mathrm{CH}), 4.03-4.17\left(\mathrm{~m}, 2 \mathrm{H}, \mathrm{CH}_{2} \mathrm{~N}\right), 7.65\left(\mathrm{~d}, J=8.5 \mathrm{~Hz}, 2 \mathrm{H}, \mathrm{H}_{\mathrm{ar}}\right)$, $7.69(\mathrm{~d}, J=15.7 \mathrm{~Hz}, 1 \mathrm{H},(\underline{\mathrm{CH}}=\mathrm{CH})), 7.85,\left(\mathrm{~d}, J=8.9 \mathrm{~Hz}, 2 \mathrm{H}, \mathrm{H}_{\mathrm{ar}}\right), 7.86\left(\mathrm{~d}, 2 \mathrm{H}, J=8.5 \mathrm{~Hz}, \mathrm{H}_{\mathrm{ar}}\right)$, $7.98(\mathrm{~d}, J=15.7 \mathrm{~Hz}, 1 \mathrm{H},(\mathrm{CH}=\underline{\mathrm{CH}})), 8.19\left(\mathrm{~d}, J=8.9 \mathrm{~Hz}, 2 \mathrm{H}, \mathrm{H}_{\mathrm{ar}}\right), 12.85(\mathrm{~s}, 1 \mathrm{H}, \mathrm{COOH}) .{ }^{13} \mathrm{C} \mathrm{NMR}$ (75 MHz, d6-DMSO) 8: 35.0 (C-4'), 35.4 (C-3'’), 49.8 (C-5'), 118.5 (C-6, 8), 122.7 (C-2), 123.9 (C-4'), 132.6 (C-4), 129.7 (C-5, 9), 130.8 (C-2', 6'), 131.9 (C-3', 5'), 134.0 (C-1'), 142.2 (C-1), 143.3 (C-7), 174.1 (C-2’”), 172.7 (COOH), 187.5 (C-3). Anal. calcd. for $\mathrm{C}_{20} \mathrm{H}_{16} \mathrm{BrNO}_{4}$, (\%): C 57.99, H 3.89, N 3.38; Found (\%), C 57.62, H 2.96, N 3.45.

1-[4-(5-Aryl-4,5-dihydro-1H-pyrazol-3-yl)phenyl]-5-oxopyrrolidine-3-carboxylic acids (4ac). General Procedure. A solution of $0.002 \mathrm{~mol}$ of the corresponding chalcones 3a-c, $0.3 \mathrm{~g}$ $(0.006 \mathrm{~mol})$ of hydrazine hydrate (concentration $99 \%)$ and $10 \mathrm{ml}$ of ethanol was refluxed for 5 hours and cooled. The precipitate filtered, and then washed with ethanol and diethyl ether.

5-Oxo-1-[4-(5-phenyl-4,5-dihydro-1 $H$-pyrazol-3-yl)phenyl]pyrrolidine-3-carboxylic acid (4a). Yield 81\%; mp 171-172 ${ }^{\circ} \mathrm{C}$ (ethanol), IR (KBr), $\mathrm{cm}^{-1}: 3419(\mathrm{OH}), 3346(\mathrm{NH}), 1679,1654$ $(\mathrm{C}=\mathrm{O}), 1606(\mathrm{C}=\mathrm{N}) . \mathrm{MS} \mathrm{m} / \mathrm{z},(\%):[\mathrm{M}+\mathrm{H}]^{+}=350.3(100) .{ }^{1} \mathrm{H}$ NMR $\left(300 \mathrm{MHz}, \mathrm{d}_{6}-\mathrm{DMSO}\right) \delta$ : 2.62-2.79 (m, 2H, $\mathrm{CH}_{2} \mathrm{CO}$ ), $2.82\left(\mathrm{dd}, J=10.7 \mathrm{~Hz}, J=16.3 \mathrm{~Hz}, 1 \mathrm{H}, \mathrm{CH}_{2}(\mathrm{C}-2)\right), 2.94-3.04$ (m, 1H, $\mathrm{CH}_{\text {pyrrolid.), }} .43$ (dd, $\left.J=10.7 \mathrm{~Hz}, J=16.3 \mathrm{~Hz}, 1 \mathrm{H}, \mathrm{CH}_{2}(\mathrm{C}-2)\right), 3.93-4.03\left(\mathrm{~m}, 2 \mathrm{H}, \mathrm{CH}_{2} \mathrm{~N}\right), 4.82$ (t, $J=10.7 \mathrm{~Hz}, 1 \mathrm{H}, \mathrm{CH}(\mathrm{C}-1)), 7.23-7.45\left(\mathrm{~m}, 5 \mathrm{H}, \mathrm{H}_{\mathrm{ar}}+\mathrm{NH}\right), 7.61,7.69\left(2 \mathrm{~d}, J=9.0 \mathrm{~Hz}, 4 \mathrm{H}, \mathrm{H}_{\mathrm{ar}}\right) .{ }^{13} \mathrm{C}$

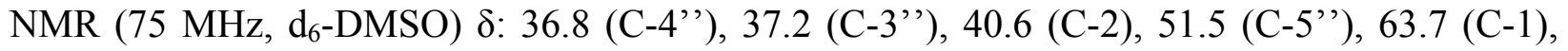
118.9 (C-6, 8), 125.8 (C-5, 9), 126.6 (C-2', 6’), 127.1 (C-4), 128.4 (C-3', 5'), 128.5 (C-4'), 139.4 (C-7), 143.0 (C-1'), $148.3(\mathrm{C}-3), 173.4(\mathrm{COOH}), 176.3\left(\mathrm{C}-2^{\prime}\right.$ '). Anal. calcd. for $\mathrm{C}_{20} \mathrm{H}_{19} \mathrm{~N}_{3} \mathrm{O}_{3}$, (\%): C 68.75, H 5.48, N 12.03; Found (\%), C 68.36, H 5.51, N 11.78.

1-\{4-[5-(4-Methoxyphenyl)-4,5-dihydro-1 $H$-pyrazol-3-yl]phenyl\}-5-oxopyrrolidine-3-carboxylic acid (4b). Yield 87\%, mp 174-175 ${ }^{\circ} \mathrm{C}$ (ethanol), IR (KBr), cm ${ }^{-1}: 3436(\mathrm{OH}), 3352(\mathrm{NH})$, 1677, $1658(\mathrm{C}=\mathrm{O}), 1607(\mathrm{C}=\mathrm{N}) . \mathrm{MS} \mathrm{m} / \mathrm{z},(\%):[\mathrm{M}+\mathrm{H}]^{+}=380.3(100) .{ }^{1} \mathrm{H}$ NMR $\left(300 \mathrm{MHz}, \mathrm{d}_{6^{-}}\right.$ DMSO) $\delta: ~ 2.62-2.78\left(\mathrm{~m}, 2 \mathrm{H}, \mathrm{CH}_{2} \mathrm{CO}\right), 2.79\left(\mathrm{dd}, J=10.7 \mathrm{~Hz}, J=16.2 \mathrm{~Hz}, 1 \mathrm{H}, \mathrm{CH}_{2}(\mathrm{C}-2)\right), 3.00-$ 3.10 (m, 1H, $\mathrm{CH}_{\text {pyrrolid.) }}, 3.38$ (dd, $\left.J=10.5 \mathrm{~Hz}, J=16.2 \mathrm{~Hz}, 1 \mathrm{H}, \mathrm{CH}_{2}(\mathrm{C}-2)\right), 3.73\left(\mathrm{~s}, 3 \mathrm{H}, \mathrm{OCH}_{3}\right)$, 3.93-4.03 (m, 2H, CH$\left.{ }_{2} \mathrm{~N}\right), 4.77(\mathrm{t}, J=10.7 \mathrm{~Hz}, 1 \mathrm{H}, \mathrm{CH}(\mathrm{C}-1)), 6.90,7.29\left(2 \mathrm{~d}, J=8.8 \mathrm{~Hz}, 4 \mathrm{H}, \mathrm{H}_{\mathrm{ar}}\right.$ ), 7.42 (br. s, $1 \mathrm{H}, \mathrm{NH}), 7.60,7.68\left(2 \mathrm{~d}, J=9.0 \mathrm{~Hz}, 4 \mathrm{H}, \mathrm{H}_{\mathrm{ar}}\right) .{ }^{13} \mathrm{C} \mathrm{NMR}\left(75 \mathrm{MHz}, \mathrm{d}_{6}\right.$-DMSO) $\delta: 36.5$ (C-4'’), 36.8 (C-3'’), 40.6 (C-2), 51.2 (C-5'’), $55.1\left(\mathrm{OCH}_{3}\right), 63.2(\mathrm{C}-1), 113.7$ (C-3', 5'), 118.9 (C-6, 8), 125.7 (C-5, 9), 127.8 (C-2', 6'), 128.7 (C-4), 134.9 (C-1'), 139.3 (C-7), 148.4 (C-3), 158.4 (C-4'), 175.7 (C-2' '), $173.1(\mathrm{COOH})$. Anal. calcd. for $\mathrm{C}_{21} \mathrm{H}_{21} \mathrm{~N}_{3} \mathrm{O}_{4},(\%)$ : C 66.48, H 5.58, N 11.07; Found (\%), C 66.12, H 5.29, N 11.25.

1-\{4-[5-(4-Bromophenyl)-4,5-dihydro-1H-pyrazol-3-yl]phenyl\}-5-oxopyrrolidine-3-carboxylic acid (4c). Yield 72\%, mp 167-168 ${ }^{\circ} \mathrm{C}$ (ethanol), IR (KBr), cm ${ }^{-1}: 3446(\mathrm{OH}), 3320(\mathrm{NH})$, 
1694, $1682(\mathrm{C}=\mathrm{O}), 1604(\mathrm{C}=\mathrm{N}) . \mathrm{MS} \mathrm{m} / \mathrm{z},(\%):[\mathrm{M}+\mathrm{H}]^{+}=428.2(100) ;[\mathrm{M}+\mathrm{H}+2]^{+}=430.2(70) .{ }^{1} \mathrm{H}$ NMR (300 MHz, d $\mathrm{d}_{6}$-DMSO) $\delta: 2.69-2.78\left(\mathrm{~m}, 2 \mathrm{H}, \mathrm{CH}_{2} \mathrm{CO}\right), 2.80(\mathrm{dd}, J=9.0 \mathrm{~Hz}, J=14.4 \mathrm{~Hz}, 1 \mathrm{H}$, $\mathrm{CH}_{2}$ (C-2)), 3.20-3.31 (m, H, CH pyrrolid.), 3,44 (dd, $J=10.6 \mathrm{~Hz}, J=16.4 \mathrm{~Hz}, 1 \mathrm{H}, \mathrm{CH}_{2}(\mathrm{C}-2)$ ), 3.95$4.11\left(\mathrm{~m}, 2 \mathrm{H}, \mathrm{CH}_{2} \mathrm{~N}\right), 4.83(\mathrm{t}, J=10,6 \mathrm{~Hz}, 1 \mathrm{H}, \mathrm{CH}(\mathrm{C}-1)), 7.18-7.94\left(\mathrm{~m}, 8 \mathrm{H}, \mathrm{H}_{\mathrm{ar}}\right) .{ }^{13} \mathrm{C}$ NMR $(75$ MHz, d6-DMSO) $\delta: 35.7$ (C-4'’), 35.7 C-3'’), 40.5 (C-2), 51.2 (C-5'’), 62.9 (C-1), 119.1 (C-6, 8), 120.1 (C-4'), 125.8 (C-5, 9), 127.1 (C-4), 128.9 (C-2', 6'), 131.2 (C-3', 5'), 139.1 (C-7), 142.5 (C-1'), 148.4 (C-3), $173.4(\mathrm{COOH}), 174.7$ (C-2'’). Anal. calcd. for $\mathrm{C}_{20} \mathrm{H}_{18} \mathrm{BrN}_{3} \mathrm{O}_{3},(\%)$ : C 56.09, H 4.24, N 9.81; Found (\%), C 56.23, H 3.96, N 10.06.

\section{1-[4-(1-Acetyl-5-aryl-4,5-dihydro-1H-pyrazol-3-yl)phenyl]-5-oxopyrrolidine-3-carboxylic} acids (5a-c). General Procedure. A solution of $0.002 \mathrm{~mol}$ of the corresponding chalcones 3a-c, $0.3 \mathrm{~g}(0.006 \mathrm{~mol})$ of hydrazine hydrate and $10 \mathrm{ml}$ of acetic acid was refluxed for 5 hours, then catalytic amount of HCI (4-5 drops) was added and yet refluxed for $30 \mathrm{~min}$. After cooling $30 \mathrm{ml}$ water was added and the resulting precipitate was filtered, washed with water.

\section{1-[4-(1-Acetyl-5-phenyl-4,5-dihydro-1 H-pyrazol-3-yl)phenyl]-5-oxopyrrolidine-3-carbo-}

xylic acid (5a).Yield 56\%, mp $247-248^{\circ} \mathrm{C}$ (ethanol), IR (KBr), $\mathrm{cm}^{-1}: 3448(\mathrm{OH}), 1730,1706$, $1655(\mathrm{C}=\mathrm{O}), 1607(\mathrm{C}=\mathrm{N}) . \mathrm{MS} \mathrm{m} / \mathrm{z},(\%):[\mathrm{M}+\mathrm{H}]^{+}=392.4(100) .{ }^{1} \mathrm{H}$ NMR $\left(300 \mathrm{MHz}, \mathrm{d}_{6}-\mathrm{DMSO}\right)$ $\delta: 2.31\left(\mathrm{~s}, 3 \mathrm{H}, \mathrm{COCH}_{3}\right), 2.69-2.87\left(\mathrm{~m}, 2 \mathrm{H}, \mathrm{CH}_{2} \mathrm{CO}\right), 3.11\left(\mathrm{dd}, J=4.5 \mathrm{~Hz}, J=18.0 \mathrm{~Hz}, 1 \mathrm{H}, \mathrm{CH}_{2}(\mathrm{C}-\right.$

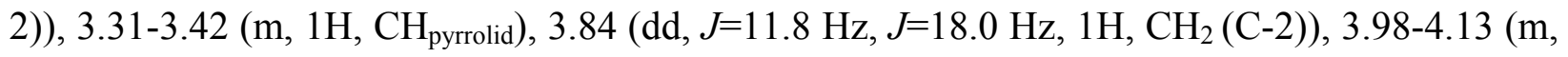
$\left.2 \mathrm{H}, \mathrm{CH}_{2} \mathrm{~N}\right), 5.53(\mathrm{dd}, J=4.5 \mathrm{~Hz}, J=11.8 \mathrm{~Hz}, 1 \mathrm{H}, \mathrm{CH}(\mathrm{C}-1)), 7.17-7.99$ (m, 10H, $\left.\mathrm{H}_{\mathrm{ar}}+\mathrm{NH}\right), 12.83$ (br. s, 1H, COOH). ${ }^{13} \mathrm{C}$ NMR (75 MHz, d 6 -DMSO) $\delta: 21.7$ (COCH 3$), 35.1$ (C4' '), 35.4 (C-3' '), 42.1 (C-2), 49.9 (C-5'’), 59.4 (C-1), 119.1 (C-6, 8), 125.4 (C-2', 6'), 126.4 (C-4), 127.2 (C-5, 9), 128.6 (C-3', 5'), 128.8 (C-4'), 140.7 (C-7), 142.5 (C-1'), 153.7 (C-3), $167.3\left(\underline{\mathrm{COCH}}_{3}\right), 172.2$ $(\mathrm{COOH}), 174.1$ (C-2' '). Anal. calcd. for $\mathrm{C}_{22} \mathrm{H}_{21} \mathrm{~N}_{3} \mathrm{O}_{4}$, (\%): C 67.51, H 5.41, N 10.74; Found (\%), C 67.45, H 5.57, N 10.53.

\section{1-\{4-[1-Acetyl-5-(4-methoxyphenyl)-4,5-dihydro-1H-pyrazol-3-yl]phenyl\}-5-oxopyrrolidine} -3-carboxylic acid (5b). Yield 84\%, mp 213-214 ${ }^{\circ} \mathrm{C}$ (2-propanol), IR (KBr), cm ${ }^{-1}: 3431(\mathrm{OH})$, 1702, 1654, $1639(\mathrm{C}=\mathrm{O}), 1608(\mathrm{C}=\mathrm{N}) . \mathrm{MS} \mathrm{m} / \mathrm{z},(\%):[\mathrm{M}+\mathrm{H}]^{+}=422.4(100) .{ }^{1} \mathrm{H} \mathrm{NMR}(300 \mathrm{MHz}$, $\mathrm{d}_{6}$-DMSO) $\delta: 2.28\left(\mathrm{~s}, 3 \mathrm{H}, \mathrm{COCH}_{3}\right), 2.70-2.86\left(\mathrm{~m}, \mathrm{CH}_{2} \mathrm{CO}\right), 3.10(\mathrm{dd}, 1 \mathrm{H}, J=4.5 \mathrm{~Hz}, J=18.0 \mathrm{~Hz}$, $1 \mathrm{H}, \mathrm{CH}_{2}(\mathrm{C}-2)$ ); 3.30-3.40 (m, 1H, $\mathrm{CH}_{\text {pyrrolid }}$ ), 3.10 (s, 3H, $\mathrm{OCH}_{3}$ ), 3.80 (dd, $J=11.7 \mathrm{~Hz}, J=18.0$ $\left.\mathrm{Hz}, 1 \mathrm{H}, \mathrm{CH}_{2}(\mathrm{C}-2)\right)$, 3.95-4.11 (m, 2H, $\left.\mathrm{CH}_{2} \mathrm{~N}\right), 5.48$ (dd, J=4.5 Hz, J=11.7 Hz, CH (C-1)), 6.87, $7.10\left(2 \mathrm{~d}, J=8,8 \mathrm{~Hz}, 4 \mathrm{H}, \mathrm{H}_{\mathrm{ar}}\right), 7.77$ (s, 4H, Har $), 11.98$ (br. s, $\left.1 \mathrm{H}, \mathrm{COOH}\right) .{ }^{13} \mathrm{C} \mathrm{NMR}\left(75 \mathrm{MHz}, \mathrm{d}_{6^{-}}\right.$

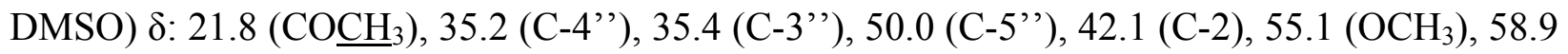
(C-1), 114.0 (C-3', 5'), 119.0 (C-6, 8), 126.5 (C-4), 126.8 (C-5, 9), 127.2 (C-2', 6'), 134.5 (C1'), 140.7 (C-7), 153.7 (C-3), 158.4 (C-4'), $167.2\left(\underline{\mathrm{COCH}}_{3}\right), 172.4$ (COOH), 174.3 (C-2'’). Anal. calcd. for $\mathrm{C}_{23} \mathrm{H}_{23} \mathrm{~N}_{3} \mathrm{O}_{5}$, (\%): C 65.55, H 5.50, N 9.97; Found (\%), C 65.29, H 5.22, N 10.21.

1-\{4-[1-Acetyl-5-(4-bromophenyl)-4,5-dihydro-1 $H$-pyrazol-3-yl]phenyl\}-5-oxopyrrolidine-

3-carboxylic acid (5c). Yield 88\%, mp 262-263 ${ }^{\circ} \mathrm{C}$ (2-propanol), IR (KBr), cm ${ }^{-1}: 3440(\mathrm{OH})$, 1704, 1662, $1652(\mathrm{C}=\mathrm{O}), \quad 1607 \quad(\mathrm{C}=\mathrm{N}) . \quad \mathrm{MS} \quad \mathrm{m} / \mathrm{z}, \quad(\%): \quad[\mathrm{M}+\mathrm{H}]^{+}=470.2(98)$, $[\mathrm{M}+\mathrm{H}+2]^{+}=472.2(100) .{ }^{1} \mathrm{H} \mathrm{NMR}\left(300 \mathrm{MHz}, \mathrm{d}_{6}-\mathrm{DMSO}\right) \delta: 2.30\left(\mathrm{~s}, 3 \mathrm{H}, \mathrm{COCH}_{3}\right), 2.68-2.86(\mathrm{~m}$, 
2H, $\mathrm{CH}_{2} \mathrm{CO}$ ), 3.13 (dd, $\left.J=4.7 \mathrm{~Hz}, J=18.1 \mathrm{~Hz}, 1 \mathrm{H}, \mathrm{CH}_{2}(\mathrm{C}-2)\right), 3.30-3.41\left(\mathrm{~m}, 1 \mathrm{H}, \mathrm{CH}_{\text {pyrrolid. }}\right)$ ), 3.83 $\left(\mathrm{dd}, J=11.8 \mathrm{~Hz}, J=18.1 \mathrm{~Hz}, 1 \mathrm{H}, \mathrm{CH}_{2}(\mathrm{C}-2)\right), 3.97-4.11\left(\mathrm{~m}, 2 \mathrm{H}, \mathrm{CH}_{2} \mathrm{~N}\right), 5.55(\mathrm{dd}, J=4.7 \mathrm{~Hz}, J=11.8$ $\mathrm{Hz}, \mathrm{CH}(\mathrm{C}-1)), 7.15,7.55$ (2d, J=8.5 Hz, 4H, Har), 7.17 (s, 4H, Har), 12.94 (br. s, 1H, COOH). ${ }^{13} \mathrm{C}$ NMR (75 MHz, d6 $\left.-\mathrm{DMSO}\right) \delta: 21.7\left(\mathrm{COCH}_{3}\right), 35.1$ (C-4') $) 35.4$ (C-3'’), 41.9 (C-2), 49.9 (C5'’), 58.8 (C-1), 119.0 (C-6, 8), 120.2 (C-4'), 126.3 (C-4), 127.3 (C-5, 9), 127.9 (C-2', 6'), 131.5 (C-3', 5'), 140.8 (C-7), 141.8 (C-1'), 153.7 (C-3), $167.4\left(\mathrm{COCH}_{3}\right), 174.3$ (C-2'’), 172.3 $(\mathrm{COOH})$. Anal. calcd. for $\mathrm{C}_{22} \mathrm{H}_{20} \mathrm{BrN}_{3} \mathrm{O}_{4}$, (\%): C 56.18, H 4.29, $\mathrm{N}$ 8.93; Found (\%), C 55.97, H 4.69, N 9.02.

1-[4-(1,5-Diaryl-4,5-dihydro-1 $H$-pyrazol-3-yl)phenyl]-5-oxopyrrolidine-3-carboxylic acids (6a-c). General Procedure. A solution of $0.003 \mathrm{~mol}$ of the corresponding chalcones 3a-c, $0.43 \mathrm{~g}$ $(0.004 \mathrm{~mol})$ of phenylhydrazine and $10 \mathrm{ml}$ of 1,4-dioxane was refluxed for 4 hours, when catalytic amount of $\mathrm{HCl}$ (4-5 drops) was added and yet refluxed for $30 \mathrm{~min}$. After cooling $50 \mathrm{ml}$ water was added and the resulting precipitate was filtered, washed with water. Purified by the repeated twice precipitation from $10 \% \mathrm{Na}_{2} \mathrm{CO}_{3}$ solution with acetic acid.

5-Oxo-1-[4-(1,5-diphenyl-4,5-dihydro-1H-pyrazol-3-yl)phenyl]pyrrolidine-3-carboxylic acid (6a). Yield 77\%, mp 160-161 ${ }^{\circ} \mathrm{C}$ (ethanol), IR (KBr), $\mathrm{cm}^{-1}: 3436(\mathrm{OH}), 1733,1705(\mathrm{C}=\mathrm{O})$, $1613(\mathrm{C}=\mathrm{N}) . \mathrm{MS} \mathrm{m} / \mathrm{z},(\%):[\mathrm{M}+\mathrm{H}]^{+}=426.5(100) .{ }^{1} \mathrm{H}$ NMR (300 MHz, $\mathrm{d}_{6}$-DMSO) $\delta: 2.74$ (br. s, $2 \mathrm{H}, \mathrm{CH}_{2} \mathrm{CO}$ ), 3.06 (dd, $J=6.2, J=17.5,1 \mathrm{H}, \mathrm{CH}_{2}(\mathrm{C}-2)$ ), 3.01 (br. s, $1 \mathrm{H}, \mathrm{CH}_{\text {pyrrolid.) }} 3.86$ (dd, $J=12.0 \mathrm{~Hz}, J=17.5 \mathrm{~Hz}, 1 \mathrm{H}, \mathrm{CH}_{2}(\mathrm{C}-2)$ ), 4.01 (br. s, $2 \mathrm{H}, \mathrm{CH}_{2} \mathrm{~N}$ ), 5.41 (dd, $1 \mathrm{H}, J=6.2 \mathrm{~Hz}, J=12.0$ $\mathrm{Hz}, \mathrm{CH}(\mathrm{C}-1)$ ), 6.67-7.92 (m, 14H, Har). ${ }^{13} \mathrm{C}$ NMR (75 MHz, d6-DMSO) $\delta: 35.2$ (C-4'”), 36.2 (C3') $), 43.6$ (C-2), 50.6 (C-5') $, 55.2\left(\mathrm{OCH}_{3}\right), 63.1$ (C-1), 112.9 (C-11, 15), 119.0 (C-13), 119.5 (C6, 8), 127.8 (C-4), 126.2 (C-5, 9), 127.4 (C-2', 6'), 128.6 (C-4'), 128.6 (C-3', 5'), 129.0 (C-12, 14), 139.6 (C-7), 142.6 (C-1'), 144.3 (C-3), 147.0 (C-10), 171.9 (COOH), 174.7 (C-2'’). Anal. calcd. for $\mathrm{C}_{26} \mathrm{H}_{23} \mathrm{~N}_{3} \mathrm{O}_{3}$, (\%): C 73.4, $\mathrm{H}$ 5.45, N 9.88; Found (\%), C 73.58, H 5.47, N 9.62.

1-\{4-[5-(4-Methoxyphenyl)-1-phenyl-4,5-dihydro-1H-pyrazol-3-yl]phenyl\}-5-oxopyrrolidine -3-carboxylic acid (6b). Yield 48\%, mp 139-140 $\mathrm{C}$ (ethanol), IR (KBr), cm ${ }^{-1}: 2931$ (OH), 1734, $1703(\mathrm{C}=\mathrm{O}), 1598(\mathrm{C}=\mathrm{N}) . \mathrm{MS} \mathrm{m} / \mathrm{z},(\%):[\mathrm{M}+\mathrm{H}]^{+}=456.3(100) .{ }^{1} \mathrm{H}$ NMR $\left(300 \mathrm{MHz}, \mathrm{d}_{6}-\mathrm{DMSO}\right)$ $\delta: 2.68-2.86\left(\mathrm{~m}, 2 \mathrm{H}, \mathrm{CH}_{2} \mathrm{CO}\right), 3.06\left(\mathrm{dd}, J=6.4 \mathrm{~Hz}, J=17,4 \mathrm{~Hz}, 1 \mathrm{H}, \mathrm{CH}_{2}(\mathrm{C}-2)\right), 3.33-3.42(\mathrm{~m}, 1 \mathrm{H}$, $\mathrm{CH}_{\text {pyrrolid.), }} 3.71$ (s, 3H, $\mathrm{OCH}_{3}$ ), 3.86 (dd, $\left.J=12.0 \mathrm{~Hz}, J=17.4 \mathrm{~Hz}, 1 \mathrm{H}, \mathrm{CH}_{2}(\mathrm{C}-2)\right), 3.97-4.11$ (m, $2 \mathrm{H}, \mathrm{CH}_{2} \mathrm{~N}$ ), 5.40 (dd, $\left.J=6.4 \mathrm{~Hz}, J=12.0 \mathrm{~Hz}, 1 \mathrm{H}, \mathrm{CH}(\mathrm{C}-1)\right), 6.70$ (t, J=7.3 Hz, 1H, $\mathrm{H}_{\mathrm{ar}}$ ), 6.89 (d, $\left.J=8.6 \mathrm{~Hz}, 2 \mathrm{H}, \mathrm{H}_{\mathrm{ar}}\right), 7.00\left(\mathrm{~d}, J=7.8 \mathrm{~Hz}, 2 \mathrm{H}, \mathrm{H}_{\mathrm{ar}}\right), 7.14\left(\mathrm{dd}, J=7.3 \mathrm{~Hz}, J=7.8 \mathrm{~Hz}, 2 \mathrm{H}, \mathrm{H}_{\mathrm{ar}}\right), 7.21$ (d, $\left.J=8.6 \mathrm{~Hz}, 2 \mathrm{H}, \mathrm{H}_{\mathrm{ar}}\right), 7.73$ (s, 4H, $\left.\mathrm{H}_{\mathrm{ar}}\right), 12.79$ (br. s. COOH). ${ }^{13} \mathrm{C}$ NMR (75 MHz, d -DMSO$^{-D} \delta$ : 35.1 (C-4"'), 35.3 (C-3'”), 43.0 (C-2), 49.9 (C-5'”), $55.0\left(\mathrm{OCH}_{3}\right), 62.6(\mathrm{C}-1), 113.0(\mathrm{C}-11,15)$, 114.3 (C-3', 5'), 118.4 (C-13), 119.2 (C-6, 8), 126.1 (C-5, 9), 127.1 (C-2', 6'), 127.9 (C-4), 128.6 (C-4'), 128.8 (C-12, 14), 134.4 (C-1'), 139.4 (C-7), 144.3 (C-3), 146.8 (C-10), 158.4 (C4'), 172.0 (COOH), 174.2 (C-2'’). Anal. calcd. for $\mathrm{C}_{27} \mathrm{H}_{25} \mathrm{~N}_{3} \mathrm{O}_{4}$, (\%): C 71.19, H 5.53, N 9.22; Found (\%), C 70.81, H 5.87, N 9.51.

1-\{4-[5-(4-Bromophenyl)-1-phenyl-4,5-dihydro-1H-pyrazol-3-yl]phenyl\}-5-oxopyrrolidine3-carboxylic acid (6c). Yield 65\%, mp 152-153 ${ }^{\circ} \mathrm{C}$ (ethanol), IR (KBr), cm ${ }^{-1}: 3027(\mathrm{OH}), 1705$, 
$1675(\mathrm{C}=\mathrm{O}), 1598(\mathrm{C}=\mathrm{N}) . \mathrm{MS} \mathrm{m} / \mathrm{z},(\%):[\mathrm{M}+\mathrm{H}]^{+}=504.3(100),[\mathrm{M}+\mathrm{H}+2]^{+}=506.3(98) .{ }^{1} \mathrm{H} \mathrm{NMR}$ (300 MHz, d $\left.\mathrm{d}_{6}-\mathrm{DMSO}\right) \delta$ : 2.69-2.86 (m, 2H, $\left.\mathrm{CH}_{2} \mathrm{CO}\right), 3.10(\mathrm{dd}, J=6.3 \mathrm{~Hz}, J=17.5 \mathrm{~Hz}, 1 \mathrm{H}$,

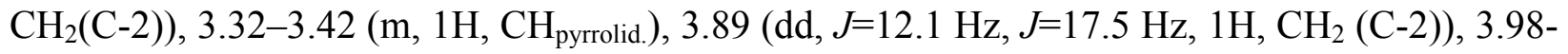
$4.11\left(\mathrm{~m}, 2 \mathrm{H}, \mathrm{CH}_{2} \mathrm{~N}\right), 5.49(\mathrm{dd}, J=6.3 \mathrm{~Hz}, J=12.1 \mathrm{~Hz}, 1 \mathrm{H}, \mathrm{CH}(\mathrm{C}-1)), 6.73\left(\mathrm{t}, J=7.3 \mathrm{~Hz}, 1 \mathrm{H}, \mathrm{H}_{\mathrm{ar}}\right)$, $6.98\left(\mathrm{~d}, J=8.5 \mathrm{~Hz}, 2 \mathrm{H}, \mathrm{H}_{\mathrm{ar}}\right), 7.16\left(\mathrm{dd}, J=7.3 \mathrm{~Hz}, J=8.5 \mathrm{~Hz}, 2 \mathrm{H}, \mathrm{H}_{\mathrm{ar}}\right), 7.25\left(\mathrm{~d}, J=8.5 \mathrm{~Hz}, 2 \mathrm{H}, \mathrm{H}_{\mathrm{ar}}\right.$ ), $7.53\left(\mathrm{~d}, J=8.5 \mathrm{~Hz}, 2 \mathrm{H}, \mathrm{H}_{\mathrm{ar}}\right), 7.74$ (s, 4H, Har), 12.91 (br. s, $\left.1 \mathrm{H}, \mathrm{COOH}\right) .{ }^{13} \mathrm{C} \mathrm{NMR}\left(75 \mathrm{MHz}, \mathrm{d}_{6^{-}}\right.$ DMSO) 8:), 35.1 (C-4'’), 35.3 (C-3'’), 42.7 (C-2), 49.9 (C-5'’), 62.4 (C-1), 112.9 (C-11, 15), 118.7 (C-13), 119.2 (C-6, 8), 120.4 (C-4'), 126.2 (C-5, 9), 127.7 (C-4), 128.2 (C-2', 6'), 129.0 (C-12, 14), 131.9 (C-3', 5'), 139.5 (C-7), 141.9 (C-1'), 144.0 (C-3), 147.0 (C-10), 172.1 $(\mathrm{COOH}), 174.2$ (C-2''). Anal. calcd. for $\mathrm{C}_{26} \mathrm{H}_{22} \mathrm{BrN}_{3} \mathrm{O}_{3}$, (\%): C 61.91, H 4.40, N 8.33; Found (\%), C 62.03, H 4.21, N 8.39.

1-[4-(1,5-Diaryl-1H-pyrazol-3-yl)phenyl]-5-oxopyrrolidine-3-carboxylic acids (7a,b). General Procedure. A solution of $0.003 \mathrm{~mol}$ of the corresponding chalcones 3a-c, $0.43 \mathrm{~g}(0.004$ mol) of phenylhydrazine and $10 \mathrm{ml}$ of acetic acid was refluxed for 4 hours, when catalytic amount of $\mathrm{HCl}$ (4-5 drops) was added and yet refluxed for $30 \mathrm{~min}$. After cooling $50 \mathrm{ml}$ water was added and the resulting precipitate was filtered, washed with water. Purified by the repeated twice precipitation from $10 \% \mathrm{Na}_{2} \mathrm{CO}_{3}$ solution with acetic acid.

5-Oxo-1-[4-(1,5-diphenyl-1H-pyrazol-3-yl)phenyl]pyrrolidine-3-carboxylic acid (7a). Yield 65\%, mp 135-136 ${ }^{\circ} \mathrm{C}$ (acetone), IR (KBr), cm ${ }^{-1}: 2908(\mathrm{OH}), 1746,1661,(\mathrm{C}=\mathrm{O}), 1609(\mathrm{C}=\mathrm{CH})$, $1597(\mathrm{C}=\mathrm{N}) . \mathrm{MS} \mathrm{m} / \mathrm{z},(\%):[\mathrm{M}+\mathrm{H}]^{+}=423.5(100) .{ }^{1} \mathrm{H} \mathrm{NMR}\left(300 \mathrm{MHz}, \mathrm{d}_{6}-\mathrm{DMSO}\right) \delta: 2.72-2.89$

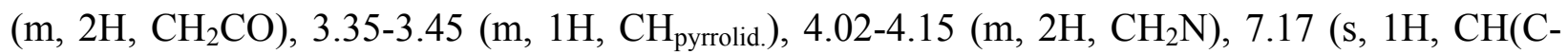
2)), 7.29-7.48 (m. 10H, $\left.\mathrm{H}_{\mathrm{ar}}\right), 7.78,7.95\left(2 \mathrm{~d}, J=8.8 \mathrm{~Hz}, 4 \mathrm{H}, \mathrm{H}_{\mathrm{ar}}\right), 12.89$ (br. s, $\left.1 \mathrm{H}, \mathrm{COOH}\right) .{ }^{13} \mathrm{C}$

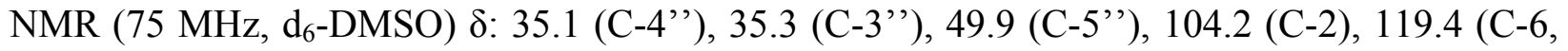
8), 125.2 (C-11, 15), 125.7 (C-5, 9), 127.7 (C-4), 128.3 (C-13), 129.3 (C-4'), 128.4 (C-3', 5'), 128.6 (C-2', 6'), 129.1 (C-12,14), 123.0 (C-1'), 138.9 (C-7), 139.8 (C-10), 144.1 (C-3), 150.5 (C-1), $171.9(\mathrm{COOH}), 174.2$ (C-2''). Anal. calcd. for $\mathrm{C}_{26} \mathrm{H}_{21} \mathrm{~N}_{3} \mathrm{O}_{3},(\%)$ : C 73.74, H 5.00, N 9.92; Found (\%), C 73.89, H 4.87, N 9.82.

1-\{4-[5-(4-Methoxyphenyl)-1-phenyl-1H-pyrazol-3-yl]phenyl\}-5-oxopyrrolidine-3-carbo-

xylic acid (7b). Yield 65\%, mp 115-116 ${ }^{\circ} \mathrm{C}$ (ethanol), IR (KBr), $\mathrm{cm}^{-1}: 3053(\mathrm{OH}), 1706,1656$ $(\mathrm{C}=\mathrm{O}), 1613(\mathrm{C}=\mathrm{CH}), 1598(\mathrm{C}=\mathrm{N}) . \mathrm{MS} \mathrm{m} / \mathrm{z},(\%):[\mathrm{M}+\mathrm{H}]^{+}=454.5(100) .{ }^{1} \mathrm{H} \mathrm{NMR}\left(300 \mathrm{MHz}, \mathrm{d}_{6^{-}}\right.$

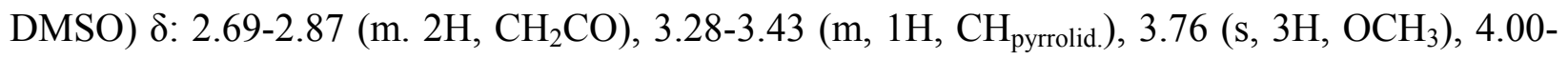
$4.14\left(\mathrm{~m}, 2 \mathrm{H}, \mathrm{CH}_{2} \mathrm{~N}\right), 6.94\left(\mathrm{~d}, J=8.9 \mathrm{~Hz}, 2 \mathrm{H}, \mathrm{H}_{\mathrm{ar}}\right), 7.07$ (s, 1H, CH (C-2)), 7.22 (d, J=8.9 Hz, 2H, $\left.\mathrm{H}_{\mathrm{ar}}\right)$, 7.32-7.47 (m, 5H, $\left.\mathrm{H}_{\mathrm{ar}}\right), 7.75,7.92\left(2 \mathrm{~d}, J=8.9 \mathrm{~Hz}, 4 \mathrm{H}, \mathrm{H}_{\mathrm{ar}}\right), 12.85$ (br. s, $\left.1 \mathrm{H}, \mathrm{COOH}\right) .{ }^{13} \mathrm{C}$ NMR (75 MHz, d 6 -DMSO) 8: 35.1 (C-4'’), 35.3 (C-3'”), 49.9 (C-5' '), $55.2\left(\mathrm{OCH}_{3}\right), 104.7$ (C-2), 114.1 (C-3', 5'), 119.4 (C-6, 8), 120.1 (C-12, 14), 122.2 (C-1'), 125.2 (C-11, 15), 125.6 (C-5, 9), 127.6 (C-4), 128.4 (C-13), 129.8 (C-2', 6'), 139.9 (C-10), 144.0 (C-3), 150.4 (C-1), 159.3 (C-4'), $171.9(\mathrm{COOH}), 174.2$ (C-2' '). Anal. calcd. for $\mathrm{C}_{27} \mathrm{H}_{23} \mathrm{~N}_{3} \mathrm{O}_{4},(\%)$ : C 71.51, H 5.11, N 9.27; Found (\%), C 71.64, H 5.36, N 8.99. 


\section{References}

1. Nehad, A.; El-Latif, Abd.; El-Galil, Abd.; Amr E.; Ibrahiem, A. A. Monatsh. Chem. 2007, $138,559$.

2. Gaede, B. J.; Mc Dermott, L. L. J. Heterocyclic Chem., 1993, 30, 49.

3. Shibata, K.; Katsuyama, I.; Izoe, H.; Matsui M.; Muramatsu H. J. Heterocyclic Chem. 1993, $30,277$.

4. Jiaxi, X; Wang, C; Zhang, Q. Heteroatom Chem. 2001, 6, 557.

5. Clinton, R.; Manson, A. J.; Stonner, F. W.; Beyler, A. L.; Potts, G. O. J. Am. Chem. Soc. 1995, 81, 1513.

6. Patra, A.; Ghosh, G.; Sengupta, P. K.; Nath, S. Magn. Reson. Chem. 1987, 25, 734.

7. Szajda, M. Magn. Reson. Chem. 1989, 27, 399.

8. Thirunarayanan, G.; Vanangamudi, G. Arkivoc 2006, (xii), 58.

9. Azarifar, D.; Ghasemnejad, H. Molecules 2003, 8, 642.

10. Levai, A.; Jekö, J. Arkivoc 2005, (x), 199.

11. Levai, A. Arkivoc 2005, (ix), 344.

12. El-Rayyes, N.; Al-Johary A. J. A. J. Chem. Eng. Data 1985, 30, 500.

13. van Hes, R.; Wellinga, K.; Grosscurt, A. C. J. Agric. Food Chem. 1978, $26,915$.

14. Shah, J. N.; Shah, C. J. Org. Chem. 1978, 43, 1266.

15. Rurack, K.; Bricks, J. L. Arkivoc 2001, (xi), 31.

16. Csunderlik, C.; Bercean, V.; Peter, F.; Badea, V. Arkivoc 2002, (ii), 133.

17. Azarifar, D.; Shaebanzadeh, M. Molecules 2002, 7, 885.

18. Figueroa, J.; Caballero, E.; Puebla, P.; Tomé, F.; Medarde, M. Molecules 2006, 11, 929.

19. Claramunt, R. M.; Escolástico, C.; Elguero, J. Arkivoc 2001, (i), 172.

20. Razus, A. C.; Birzan, L.; Nae, S.; Lehadus, O. L.; Pavel, C.; Costan, O. Arkivoc 2005, (x), 71.

21. Jędrysiak, R.; Sawicki, M.; Wagner, P.; Suwinski, J. Arkivoc 2007, (vi), 103.

22. Farghaly, A. R.; El-Kashef, H. Arkivoc 2006, (xi), 76.

23. Katritzky, A.; Vakulenko, A. V.; Akue-Gedu, R.; Gromova, A.V.; Witek, R.; Roger, J. W. Arkivoc 2007, (i), 9.

24. Abdelnamid, A. O.; Zohdi, H, F.; Sallam, M. M. M.; Ahmed, N. A. Molecules 2000, 5, 967.

25. Prakash, O.; Pannu, K. Arkivoc 2007, (xiii), 28.

26. Babbitt, G.; Lynch, M. P.; Beck, J. R. Magn. Reson. Chem. 1990, 31(1), 90.

27. Martins, M. A. P.; Zanatta, N.; Bonacorso, H. G.; Rosa, A. F.; Claramunt, R. M.; Garcia, M. A.; Maria, M. D. S.; Elguero, J. Arkivoc 2006, (iv), 29.

28. Dumitraşcu, F.; Mitan, C. I.; Dumitrescu, D.; Drăghici, C.; Căproiu, M. T. Arkivoc 2002, (ii), 80.

29. Foces-Foces, C.; Infantes, L.; Aguilar-Parrilla, F.; Golubev, N. S.; Limbach, H.-H.; Elguero, J. J. Chem. Soc. Perkin Trans 2 1996, 1, 349.

30. Rykowski, A.; Wolinska, E.; Branowska, D.; Van der Plas, H. C. Arkivoc 2004, (iii), 74. 
31. Bruix, M.; de Mendoza, J.; Claramunt, R, M.; Elguero, J. Magn. Reson. Chem. 1985, 23, 367.

32. Bouabdallah, I.; Tauzani, R.; Zidane, I.; Ramdani, A.; Radi, S. Arkivoc 2006, (xiv), 46.

33. Bouabdallah, I.; Tauzani, R.; Zidane, I; Ramdani, A.; Radi, S. Arkivoc 2006, (xii), 138.

34. Brokaite, K.; Mickevičius, V.; Mikulskienè G. Khim. Geterotsikl. Soedin. 2006, 9, 1336. 